International Journal of Pattern Recognition and Artificial Intelligence

Vol. 31, No. 7 (2017) 1759013 (21 pages)

(C) The Author(s)

DOI: $10.1142 / \mathrm{S} 0218001417590133$

\title{
A Load-Balancing Energy Consumption Minimization Scheme in 5G Heterogeneous Small Cell Wireless Networks Under Coverage Probability Analysis
}

\author{
Zhu Xiao, ${ }^{*, \uparrow}$, Shuangchun $\mathrm{Li}^{*, \|}$, Xiaochun Chen ${ }^{*, * *}$, \\ Dong Wang*, ${ }^{* \dagger}$ and Wenjie Chen ${ }^{\S}$, \\ ${ }^{*}$ College of Computer Science and Electronic Engineering \\ Hunan University, Changsha 410082, P. R. China \\ ${ }^{\dagger}$ State Key Laboratory of Integrated Services Networks \\ Xidian University, Xian 710071, P. R. China \\ *Law School, Hunan University, Changsha, 410082, P. R. China \\ $\S_{\text {Business College, Central South University of Forestry and Technology }}$ \\ Changsha 410004, P. R. China \\ 『zxiao@hnu.edu.cn \\ "lishuangchun@hnu.edu.cn \\ **chenxiaochun1601@vip.163.com \\ ${ }^{\dagger}$ wangd@hnu.edu.cn \\ *wendychen711@126.com
}

Received 1 October 2016

Accepted 21 December 2016

Published 3 March 2017

Heterogeneous small cell networks (HSCN), as a promising paradigm to increase end-user data rates and improve the overall capacity, is expected to be a key cellular architecture in $5 \mathrm{G}$ wireless networks. However, energy consumed in HSCN is considerable due to the massive use of small cells. In this paper, we investigate the energy consumption issue which stems from the enormous number of running small cell base stations (SBSs) deploying in the HSCN. We first propose power consumption models so as to characterize the active state and the idle state of SBSs, respectively. Then two sleep modes for SBSs tier, i.e. random sleep mode and load-awareness dynamic sleep mode, are proposed. The random sleep is designed based on a binomial distribution of the SBS operation probability. Through the analysis on activeness of SBSs, we define the operation probability for the SBS applying the proposed dynamic sleep mode is associated to its traffic load level. The closed-form expressions of success probability for coverage, which is used to decide whether an active user can connect to a SBS successfully, are derived for the proposed sleep modes. Energy consumption minimizations are presented for the two proposed sleep modes under the success probability constraint. Simulation results prove the effectiveness of the proposed two sleep modes. Different energy saving gains can be achieved via using of the energy saving strategy.

\$t Corresponding author.

This is an Open Access article published by World Scientific Publishing Company. It is distributed under the terms of the Creative Commons Attribution 4.0 (CC-BY) License. Further distribution of this work is permitted, provided the original work is properly cited. 
The superior of the dynamic sleep mode by comparing the random sleep is also verified in terms of energy consumption, success probability and power efficiency.

Keywords: Small cell; energy consumption; power efficiency; sleep mode; heterogeneous networks.

\section{Introduction}

For more than ten years into the 21st century, along with the gradual rise of mobile Internet, the successful application of cloud services, the proliferation of smart mobile phones and a variety of heterogeneous computing devices, ${ }^{23}$ the demand for mobile data has begun to grow explosively. ${ }^{19}$ According to the prediction and statistical analysis from Cisco and Qualcomm, ${ }^{3,13}$ the global mobile data will continue exponential growth in the next decade, that is, during the years 2015-2025, mobile data demand will appear to increase 1000 times. This requires a new wireless access technology which has the ability to expand current network system capacity to 1000 times. Undoubtedly, it will be a huge challenge to the current mobile communication system and wireless communication network. ${ }^{20}$

To alleviate the above problem, a method for promoting the system performance is to consider the topological structure of the wireless network, i.e. the cellular network isomerization and cell miniaturization ${ }^{14}$ : to build hyper dense heterogeneous small cells in existing macro cellular network, by means of the massive use of various types of small cells, including microcell, picocell, femtocell working in the licensed frequency band and Wi-Fi working in the nonauthorized frequency band, which are deployed densely within the coverage range of the macrocell base station (MBS) to enhance the system capacity and improve the data rate. ${ }^{9}$ This is the concept of heterogeneous small cell network put forward in recent years. The small cell tier can share most of the communication service, and provide the wireless communication service with a relatively high data rate to the near mobile user (MU) terminal. This heterogeneous communication network structure is regarded as a breakthrough to the traditional communication network structure. The former greatly improves space utilization rate of spectrum, high data rate, low cost, easy maintenance and deployment flexibility. ${ }^{10}$

The heterogeneous small cell networks (HSCN), which comprise a conventional macrocell network overlaid with a diverse set of small cells including picocells and femtocells, ${ }^{18}$ are expected to be the key cellular architecture of $5 \mathrm{G}$ for provisioning better quality of service and higher end-user data rates. ${ }^{17}$ Each small cell itself consumes less energy than the MBS. However, the number of the small cells deployed in a certain area surprisingly may be in quantities of thousands. Therefore, the enormous number of running small cell base stations (SBSs) leads to energy consumed in the whole cellular network rising to a whopping value. This in turn causes considerably increase in the cellular network operation burden. In this context, the problem of how to reduce energy consumption and improve energy efficiency is worth pondering due to the excessive energy consumption and energy inefficiency being one of the main causes leading to a large operational cost. 


\subsection{Literature review}

In order to solve the energy consumption problem, the concept of sleep strategy has been proposed and applied in the SBS tier in recent years. The motivation behind this can be explained by the higher volatility in traffic demand over space and time in cellular networks, which has been confirmed in Ref. 7. In the existing cellular networks, it is usually assumed that the traffic demand is always staying at a high level and that the networks are in operation $24 \mathrm{~h}$ a day. Energy conservation by virtue of the sleep mode for wireless communication terminal running on battery power has been studied in Ref. 5. Sleep mode supported by IEEE 802.16e and LTE standards has been investigated in Ref. 16. In Ref. 2, based on trade-off between energy and traffic, an operation mechanism for the base station is proposed and a greedy algorithm is proposed which improves the energy efficiency via enabling certain base stations to switch between on and off states. However, in Ref. 6, the effect of sleep mode control operation mechanisms on the MU performance is negligent due to its significance on evaluating the availability of sleep mode. In Ref. 11, clustering based power saving algorithm for sleep mode is proposed in femtocell networks to keep femtocells in the energy saving mode while preserving the quality of service (QoS) experienced by users. An opportunistic way for sleep mechanism is presented in Ref. 15 to optimize energy efficiency for current conventional cellular networks and a significant conclusion is drawn so that the effectiveness bought by using sleep mode can be obtained when the cell size is small and the traffic within the SBS is light. The authors in Ref. 1 designed a class of energy saving sleep mode for the SBS tier, which allowed hardware components in the SBS to be astutely switched off in the sleep condition. And three different sleep algorithms are discussed, corresponding to small cell driven, core network driven, and user equipment driven approaches, respectively. Finally, Ref. 1 concludes that various sleep modes can achieve different energy gains in the aspect of energy consumption according to the concrete sleep policy utilized in the small cell tier. In a word, how to explore the relationship between energy consumption and evaluation parameters based on an analytical model, and optimize energy efficiency of the heterogeneous cellular network, such as QoS, communication traffic, MU data transmission rate, is still an open research topic.

\subsection{Objectives and contributions}

In this paper, we take advantage of theoretical knowledge from stochastic geometry to study the energy saving strategy for the heterogeneous small cell networks (HSCN). We first present the system model for dense SBS deploying in the macrocell. Spatial distribution of the SBS is modeled as a Homogeneous Poisson Point Process (HPPP) because the current literature has shown that using HPPP can better approximate reality than traditional hexagonal cellular model. We also formulate two power consumption models for two states of small cells, i.e. the active state and idle state, respectively. Next, we propose two sleep modes, random sleep mode and loadawareness dynamic sleep mode for small cells, with purpose of reducing energy 
consumption and improving power efficiency. We derive the optimal trade-off between energy and some evaluation parameter values by minimizing energy consumption under QoS constraints, such as coverage probability. Identifying the optimal operation probability scope for the small cell is also our primary target in the case of sleep mode. Finally, based on results obtained from analysis and simulations, an optimal operation scheme relying on sleep mode for small cells is proposed. The contributions of our work can be summarized as follows:

(i) The random sleep is modeled on the basis of a binomial distribution of the SBS operation probability, wherein each individual SBS operates with a certain probability $q$ and sleeps with $(1-q)$ when applying the random sleep mode. On the other hand, the load-awareness dynamic sleep is set to associate with the traffic load level measured by SBS. The SBS configured with the proposed dynamic sleep operates with probability $s(x)$ and sleeps with $1-s(x)$ if it has the traffic load level $x$, in which $s(x)$ is defined as a piecewise function that depends on the traffic load level $x$.

(ii) We investigate the success probability that is used to decide the possibility, if an active MU connects to an awaked SBS successfully. The closed-form expression of success probability is derived for each of the proposed two sleep modes. Energy consumption minimization is presented based on the success probability constraint. Simulation results confirm the effectiveness of the proposed sleep modes in terms of reduction on energy consumption and improvement on power efficiency.

The paper is structured as follows. Section 2 establishes the system model. Section 3 describes the power consumption model for the SBSs. Section 4 presents the proposed sleep modes. Section 5 investigates the energy minimization for the SBS tier via the study of success probability. Section 6 demonstrates the simulation results. Finally, Sec. 6 concludes the paper.

\section{System Model for Small Cell Deployment}

A hyper dense heterogeneous cellular network model is considered, which is composed of a macro cell tier and a small cell tier. Macro cell base stations (MBSs) which are used to guarantee widely signal coverage obeys the HPPP of density $\lambda_{m}$. In our study, we select separated spectrum allocation between small cell tier and macro tier, namely, the small cells use the dedicated frequency spectrum band. Hence, we do not consider the interference from the macro tier. The small cell tier is made up of a large number of SBSs whose deployment position are intensive and random, these SBSs are located according to HPPP of density $\lambda_{s}$, as shown in Fig. 1. The small cells are running on the open access to provide the cellular network mobile users with higher data rate, better QoS and larger traffic demand. According to Campbell's theory, the average number of small cells within each of micro Poisson-Voronio cells is $\frac{\lambda_{m}}{\lambda_{s}}$. ${ }^{4}$ The cellular network MUs scattered about the plane fulfills a specific random geometric distribution, for example a uniform distribution. Each active user connects with the nearest small cell to enjoy the communication service while all other base stations act as interferers. We assume that these small cells within a macro cell 


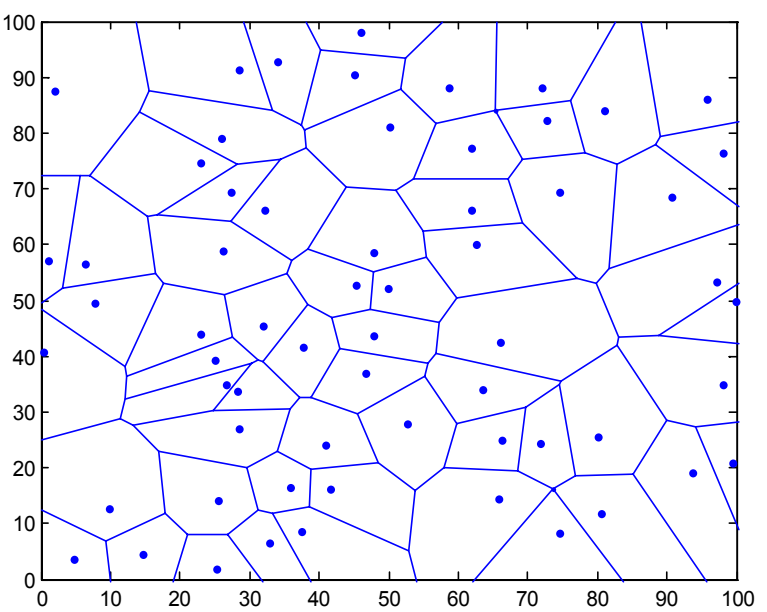

Fig. 1. HPPP distributed SBSs within an area of $10 \mathrm{~km}^{2}$.

coverage share spectrum resource whose bandwidth is. The general frequency reuse is used among all of the SBSs. When there are multiple active users in a small cell coverage range, it performs orthogonal resource sharing, such as TDMA or FDMA.

\section{Power Consumption Model}

A thorough understanding for the small cell base station hardware composition and principle is of great importance to sleep mode design because the sleep mode can make full use of the switching-off of certain hardware components in low traffic condition. According to the hardware design for small cell described in Ref. 1, the small cell base station hardware structure consists mainly of three units working together, as illustrated in Fig. 2. The first unit is comprised of a microprocessor with associated memory module which is responsible for implementation and management of standardized radio protocols as well as associated base band processing. Furthermore, it is also in charge of managing backhaul connection to the core network. These capabilities are implemented as a heterogeneous multi-core application-specific integrated circuit which has the additional benefit of low power consumption. ${ }^{8,12}$ One or more on-chip memory and random access memory components required for various data handling functions and system boot up are connected to the microprocessor. ${ }^{21,22}$ The second unit consists of a power amplifier, radio frequency receiver and radio frequency transmitter, which are mainly responsible for signal transmitting and receiving. Each component in the second unit consumes a certain amount of power. The power amplifier with cost efficiency, high linearity and gain is required to reduce cost implications in small cell hardware design, which is present to amplify signal and then pass the high power signal to the transmitting antenna. The third unit is the Field Programmable Gate Array (FPGA) with other integrated circuitry to support a series of functions including data encryption, 


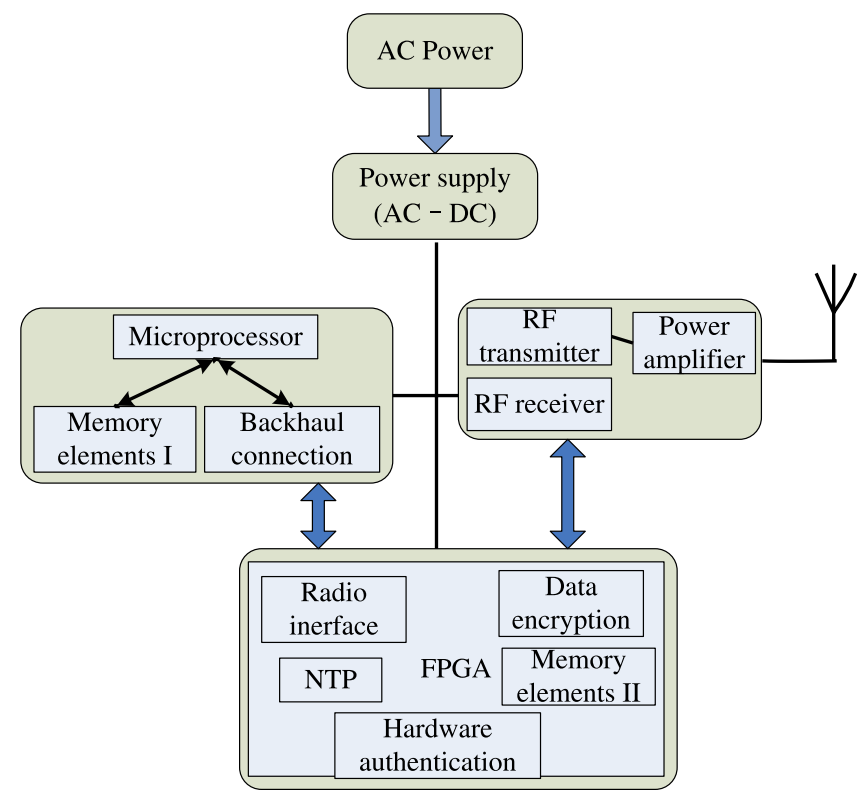

Fig. 2. Typical hardware model for a SBS.

hardware authentication, network time protocol and other host features. The radio component within the FPGA is used to be an interface between the microprocessor and the radio frequency transceiver. Due to the existence of an FPGA in the small cell hardware design, the much needed flexibility for integrating small cell hardware proprietary solutions can be achieved easier.

\subsection{Power consumption model for $S B S$}

According to the study on SBS hardware composition and principle in Ref. 1, we formulate the power consumption models for the SBS aiming at describing its active state as well as idle state. The power consumption corresponds to each hardware component in the SBS is summarized in Table 1.

Table 1. Power for each hardware component of SBS.

\begin{tabular}{lc}
\hline Hardware component & Power consumption $(\mathrm{W})$ \\
\hline Microprocessor & $P_{\mathrm{MP}}=1.7$ \\
Associated memory I & $P_{\mathrm{AMI}}=0.5$ \\
Backhaul Circuitry & $P_{\mathrm{BC}}=0.5$ \\
FPGA & $P_{\mathrm{FPGA}}=2.0$ \\
Associated memory II & $P_{\mathrm{AMII}}=0.5$ \\
Other hardware functions & $P_{\mathrm{OHF}}=1.5$ \\
RF transmitter & $P_{\mathrm{T}}=1.0$ \\
RF receiver & $P_{\mathrm{R}}=0.5$ \\
RF power amplifier & $P_{\mathrm{PA}}=2.0$ \\
\hline
\end{tabular}




\subsubsection{Power consumption model for active state}

When the SBS is with active (operation) state, all the hardware components are fully switched on. Therefore, according to Table 1, the total operation power $P_{\text {on }}$ for an active SBS can be expressed as

$$
P_{\mathrm{on}}=P_{\mathrm{MP}}+P_{\mathrm{AMI}}+P_{\mathrm{BC}}+P_{\mathrm{FPGA}}+P_{\mathrm{AMII}}+P_{\mathrm{OHF}}+P_{\mathrm{T}}+P_{\mathrm{R}}+P_{\mathrm{PA}},
$$

where $P_{\mathrm{MP}}, P_{\mathrm{AMI}}, P_{\mathrm{BC}}, P_{\mathrm{FPGA}}, P_{\mathrm{AMII}}, P_{\mathrm{OHF}}, P_{\mathrm{T}}, P_{\mathrm{R}}$ and $P_{\mathrm{PA}}$ represents the power consumption of microprocessor, associated memory I, backhaul circuitry, FPGA, associated memory II, other hardware functions, RF receiver, transmitter, RF receiver and RF power amplifier, respectively.

\subsubsection{Power consumption model for idle state}

The SBS can switch off certain hardware components when it tends to be with idle state, i.e. before entering the sleep mode. Then, the SBS is correspondingly said to reside in the idle state. Most of the hardware components presented in Table 1 can be switched off, while a few of devices should be turned on, in order to provide the backhaul circuitry to access core network and maintain signal detection during idle state. Therefore, SBS with such status essentially behaves as signal detector, which has a low-power sniffer capability that allows detecting uplink signal to sense calls from an active MU. Let $P_{0}=1 \mathrm{~W}$ denotes the static power consumed by the SBS in idle state. Let $P_{B C}$ denote the power consumption of the backhaul, the total power consumed by a SBS in the idle state can be given by

$$
P_{\text {sleep }}=P_{\mathrm{BC}}+P_{0} \text {. }
$$

\subsection{SINR analysis}

Without loss of generality, the SINR threshold $T$ is set for SBS in order to guarantee the service quality of the small cells. The MU is associated with the service base station if SINR is higher than $T$, which means that the user can access the small cell successfully. Hence, when a MU can receive signals from several SBSs and their received SINR are larger than $T$, the MU chooses the nearest one as the serving SBS. The interference power $I_{r}$ is the aggregation of the received power from all interfering SBSs. There is no cell internal interference because the small cell performs orthogonal resource sharing when there are multiple users in a small cell. Since the distribution of small cells is a stationary process, the parameter $r$ is used to denote the distance between a MU and its serving SBS. And the distribution of stays unchanged regardless of the exact user locations. The probability density function (PDF) for distance $r$ can be given as

$$
f(r)=2 \pi \lambda_{s} r \exp \left(-\pi \lambda_{s} r^{2}\right) .
$$

The set $\Phi$ consists of a set of SBSs, which is expressed as $\Phi=\left\{1,2 \ldots k \ldots \frac{\lambda_{s}}{\lambda_{m}}\right\}$. The noise is assumed to be additive white Gaussian noise (AWGN). Let $\sigma^{2}$ denote the 
noise power. The received power at the active mobile use terminal from the $k$ th SBS at the distance $r$ can be expressed as $P_{T} h r^{-\alpha}$. The parameter $h$ following the exponential distribution with argument $\mu$ is the channel fading power gain. Therefore, the SINR at the MU terminal received from the associated SBS $k$ at the distance $r$ can be expressed as

$$
\begin{aligned}
\mathrm{SINR} & =\frac{P_{T} h r^{-\alpha}}{I_{r}+\sigma^{2}}, \\
I_{r} & =\sum_{i \in \Phi \backslash k} g_{i} R_{i}^{-\alpha},
\end{aligned}
$$

where, the set $\Phi$ consists of all interfering SBSs. Path loss exponent $\alpha>2$ is from the standard power loss propagation model. The parameter $R_{i}$ represents the distance from the considered MU to the $i$ th interfering SBS. We can assume $R_{i}>r$. The interference power $I_{r}$ has channel fading value $g_{i}$, which is assumed to have same statistical distribution with $h$.

\section{Energy Saving Strategies Using Random Sleep and Dynamic Sleep for Small Cells}

The SBS enters into the low power sleep mode to save energy through switching off its certain hardware components in some circumstances, (i) there is no active users in quite a number of SBSs for a long period of time when we take into account that the active users occurrence within a SBS coverage is a random event; (ii) when traffic demand is relatively low in some SBSs coverage range during a specific time period, especially in the later half of the night, the MBS is able to provide the low traffic demand.

Therefore, in this section, two sleep modes are proposed for the small cell tier, which can be called random sleep and dynamic sleep separately. The power consumed by a sleeping SBS is set to $P_{\text {sleep }}$. On the other hand, to maintain similar network coverage and at the same time to avoid coverage holes after some small cells have been brought into sleep state, we assume that each awaked SBS transmits with power $\beta P_{T}$. The parameter $\beta$ is the power control parameter. That is to say, the addend $\beta P_{T}$ denotes the uniform increase in transmission power for small cell ratio frequency transmitter. It is assumed that $\beta$ is same for all SBSs in different sleep modes.

\subsection{Random sleep mode}

The random sleep is modeled based on binomial distribution of the operation probability. Operation means that a SBS is active. Therefore, in the random sleep mode, we can define an active SBS operates with probability $q_{\mathrm{on}}$. As a result, the $i$ th $\left(i \in\left[0, \frac{\lambda_{s}}{\lambda_{m}}\right]\right)$ SBS operates with probability $q_{\text {on }}^{i}$ and sleeps with probability 
$q_{\text {sleep }}^{i}=1-q_{\mathrm{on}}^{i}$, independent of all other SBSs. Hence, the total power $P_{\mathrm{RS}}$ consumed by the SBS tier after applying the proposed random sleep is given by

$$
P_{\mathrm{RS}}=\sum_{i=0}^{\frac{\lambda_{s}}{\lambda_{m}}}\left[q_{\text {on }}^{i}\left(P_{\text {on }}+\beta P_{T}\right)+q_{\text {sleep }}^{i} P_{\text {sleep }}\right] .
$$

\subsection{Load-awareness dynamic sleep mode}

Instead of randomly switching off SBSs, each SBS executes load-awareness dynamic sleep according to its load level. That is why this type of sleep pattern is called Loadawareness dynamic sleep. For a SBS has load level $x$, we give a function $s(x)$ to describe the operation probability. Assume that the $i$ th SBS has load level $x_{i}$, then it operates with probability $s\left(x_{i}\right)$ and sleeps with $1-s\left(x_{i}\right)$, and it is noticed that is a function of the SBS load level $s\left(x_{i}\right)$ and $s\left(x_{i}\right) \in[0,1]$. Moreover, the slope of $s\left(x_{i}\right)$ becomes larger with the increasing of load level $x_{i}$ within limits. Hence, the operation probability $s\left(x_{i}\right)$ can be defined a piecewise function which keeps growing rapidly when the load level $x_{i}$ increases closer to the threshold $x_{0}$. In addition, $s\left(x_{i}\right)$ maintains a constant value once $x_{i} \geq x_{0}$. The predefined $x_{0}$ is the load level threshold which is used to control status transition of a SBS between operation with probability and certainty. Therefore, the operation probability for the $i$ th SBS represented by $s\left(x_{i}\right)$ can be defined as follows:

$$
s\left(x_{i}\right)= \begin{cases}0 & x_{i}=0 \\ e^{\left(x_{i}-x_{0}\right)^{n}} & 0<x_{i}<x_{0}, \quad n \in N^{*} \\ 1 & x_{i} \geq x_{0}\end{cases}
$$

where, $n$ is used to control the SBS activeness sensitivity. It is assumed that the load level threshold $x_{0}$ is same for all SBSs. We assume the SBS can automatically compute its load level within its coverage area via certain technological means prewired in it so that the load level threshold $x_{0}$ can be obtained in terms of traffic demand in specific time period. Hence, in our study, we no longer care about how to calculate the load level for the small cell. As a result, the total power consumption $P_{\mathrm{DS}}$ for the SBS tier after employing the proposed load-awareness dynamic sleep mode can be given by

$$
P_{\mathrm{DS}}=\sum_{i=0}^{\frac{\lambda_{s}}{\lambda_{m}}}\left\{s\left(x_{i}\right)\left(P_{\text {on }}+\beta P_{T}\right)+\left[1-s\left(x_{i}\right)\right] P_{\text {sleep }}\right\} .
$$

\section{Energy Consumption Minimization via Success Probability Constraint}

In this section, we explored the effect on deploying the SBSs with the proposed two sleep policies, including that the power efficiency and the success probability are derived to act as our performance measures which are used to judge the effectiveness 
of the two sleep modes. Also, the noise here is considered as AWGN for the sake of simplicity. The recent work has shown that the improvement in received signal power by adding more small cells and bringing the transmitters closer to the receivers is equally canceled out by the increased interference from more small cells (interferers) such that the AWGN can be ignored. Note that the negative effect bought by AWGN should not be ignored when we deploy sleep technology in these small cells, because the successful application of sleep mode in the small cell tier can lead to the number of interferers decreased significantly. Hence, we configure the power $\sigma^{2}>0$ for AWGN.

Finally, energy consumption minimization is formulated and derived in order to explore the effectiveness of the proposed two sleep modes.

\subsection{Success probability}

The success probability can be defined as

$$
q_{S}=E[P(\operatorname{SINR}>T)] .
$$

The above expression stands for the coverage probability that can be calculated as mathematical expectation about the probability of SINR $>T$. T is referred to as the threshold. Therefore, from this perspective, the coverage probability can be also perceived as the success probability which represents the size of possibility of an active MU connecting to the active SBS at the distance successfully. Therefore, simply, the success probability can be regarded as the possibility of an active MU connecting to an awaked SBS successfully.

\subsubsection{Success probability for random sleep}

The proposed random sleep is modeled as a binomial distribution of the SBS operation probability. For the sake of simplicity, the operation probability takes the same value for all SBSs. Hence, the success probability $q_{s}^{\mathrm{RS}}$ of a randomly located user under the random sleep is given by

$$
\begin{aligned}
q_{S}^{\mathrm{RS}}= & 2 \pi \lambda_{s} q \int_{r>0} r \cdot \exp \left[-\pi \lambda_{s} q r^{2}\left(1+T^{\frac{2}{\alpha}} \int_{T^{-\frac{2}{\alpha}>0}} \frac{1}{1+u^{\frac{\alpha}{2}}} d u\right)\right] \\
& \cdot \exp \left(-\mu T r^{\alpha} \sigma^{2}\right) d r .
\end{aligned}
$$

Proof. The coverage probability $q_{S}^{\mathrm{RS}}$ related to random sleep can be deduced as following.

$$
\begin{aligned}
& q_{S}^{\mathrm{RS}}=E[P(\operatorname{SINR}>T)] \\
& =E\left[P\left(\frac{P_{T} h r^{-\alpha}}{I_{r}+\sigma^{2}}>T\right)\right], \\
& \stackrel{P_{T}=1 W}{=} E\left\{P\left[h>\operatorname{Tr}^{\alpha}\left(\sigma^{2}+I_{r}\right)\right]\right\}
\end{aligned}
$$


where the identifier $E$ represents mathematical expectation. The random variable $h$ obeys exponential distribution with intensity $\mu$ such that it can get the following equation.

$$
\left\{P\left[h>\operatorname{Tr}^{\alpha}\left(\sigma^{2}+I_{r}\right)\right]\right\}=E\left(e^{-\mu \operatorname{Tr}^{\alpha} \sigma^{2}} \cdot e^{-\mu \operatorname{Tr}^{\alpha} I_{r}}\right) .
$$

Because $r$ and $I_{r}$ are all random variables. Moreover, both are independent of each other. According to the mathematical expectation of bivariate continuous random variable and integration theory, the following expression can be obtained.

$$
\begin{aligned}
E\left(e^{-\mu T r^{\alpha} \sigma^{2}} \cdot e^{-\mu \operatorname{Tr}^{\alpha} I_{r}}\right) & =\int_{r>0} d r \int_{I_{r}} e^{-\mu \operatorname{Tr}^{\alpha} \sigma^{2}} \cdot e^{-\mu \operatorname{Tr}^{\alpha} I_{r}} f(r) \cdot f\left(I_{r}\right) d I_{r} \\
& =\int_{r>0} e^{-\mu T r^{\alpha} \sigma^{2}} \cdot 2 \pi \lambda_{s} q r \cdot e^{-\pi \lambda_{s} q r^{2}} d r \int_{I_{r}} f\left(I_{r}\right) e^{-\mu T r^{\alpha} I_{r}} d I_{r} \\
& =\left.\int_{r>0} e^{-\mu T r^{\alpha} \sigma^{2}} \cdot 2 \pi \lambda_{s} q r \cdot e^{-\pi \lambda_{s} q r^{2}} \cdot F_{I_{r}}(s)\right|_{s=\mu T r^{\alpha}} d r
\end{aligned}
$$

where the Laplace transform for the random variable $I_{r}$ is $F_{I_{r}}(s)=$ $\int_{I_{r}} f\left(I_{r}\right) e^{-s I_{r}} d I_{r}$, thus we have

$$
\int_{I_{r}} f\left(I_{r}\right) e^{-\mu T r^{\alpha} I_{r}} d I_{r}=\left.F_{I_{r}}(s)\right|_{s=\mu T r^{\alpha}} .
$$

When the random variable $g_{i}$ follows the same distribution with $h$ and $g_{i}(i=$ $1,2, \ldots)$ are independent identically distributed. Thus, the PDF for $g_{i}$ can be given.

$$
f\left(g_{i}\right)= \begin{cases}\mu e^{-\mu g_{i}} & g_{i}>0 \\ 0 & g_{i} \leq 0\end{cases}
$$

Then, the following formula can be attained.

$$
\begin{aligned}
F_{I_{r}}(s) & =E_{\Phi,\left\{g_{i}\right\}}\left\{\prod_{i \in \Phi \backslash k} E_{g_{i}}\left(e^{\left.-s g_{i} R_{i}^{-\alpha}\right)}\right\}\right. \\
& =E_{\Phi}\left\{\prod_{i \in \Phi \backslash k} \int_{-\infty}^{+\infty} e^{-s g_{i} R_{i}^{-\alpha}} \cdot f\left(g_{i}\right) d g_{i}\right\} \\
& =E_{\Phi}\left\{\prod_{i \in \Phi \backslash k} \int_{0}^{+\infty} e^{-s g_{i} R_{i}^{-\alpha}} \cdot \mu e^{-\mu g_{i}} d g_{i}\right\} \\
& =E_{\Phi}\left[\prod_{i \in \Phi \backslash k}\left(\mu \int_{0}^{+\infty} e^{-\left(s R_{i}^{-\alpha}+\mu\right) g_{i}} d g_{i}\right)\right]
\end{aligned}
$$


Z. Xiao et al.

$$
\begin{aligned}
& =E_{\Phi}\left[\prod_{i \in \Phi \backslash k} \frac{\mu}{\mu+s R_{i}^{-\alpha}}\right] \\
& =\exp \left[-2 \pi q \lambda_{s} \int_{r>0}\left(1-\frac{\mu}{\mu+s v^{-\alpha}}\right) v d v\right]
\end{aligned}
$$

Then, by using $s=\mu \operatorname{Tr}^{\alpha}$ into $F_{I_{r}}(s)$, we immediately get the following expression.

$$
\begin{gathered}
\left.F_{I_{r}}(s)\right|_{s=\mu T r^{\alpha}}=\exp \left[-2 \pi \lambda_{s} \int_{r>0} \frac{T}{T+\left(\frac{\mathrm{v}}{r}\right)^{\alpha}} v d v\right] \\
u=\left(\frac{v}{\underline{\underline{r}} \underline{T}^{\frac{1}{\alpha}}}\right)^{2} \exp \left[-\pi r^{2} \lambda_{s} \cdot T^{\frac{2}{\alpha}} \int_{T^{-\frac{2}{\alpha}>0}} \frac{1}{1+u^{\frac{\alpha}{2}}} d u\right] .
\end{gathered}
$$

Finally, the coverage probability can be calculated as following.

$$
\begin{aligned}
q_{S}^{\mathrm{RS}}= & \left.\int_{r>0} e^{-\mu \operatorname{Tr}^{\alpha} \sigma^{2}} \cdot 2 \pi \lambda_{s} q r \cdot e^{-\pi \lambda_{s} q r^{2}} \cdot F(s)\right|_{s=\mu T r^{\alpha}} d r \\
= & \int_{r>0} e^{-\mu T r^{\alpha} \sigma^{2}} \cdot 2 \pi \lambda_{s} q r \cdot e^{-\pi \lambda_{s} q r^{2}} \cdot \exp \left[-\pi r^{2} \lambda_{s} \cdot T^{\frac{2}{\alpha}} \int_{T^{-\frac{2}{\alpha}>0}} \frac{1}{1+u^{\frac{\alpha}{2}}} d u\right] d r \\
= & 2 \pi \lambda_{s} q \int_{r>0} r \cdot \exp \left[-\pi \lambda_{s} q r^{2}\left(1+T^{\frac{2}{\alpha}} \int_{T^{-\frac{2}{\alpha}>0}} \frac{1}{1+u^{\frac{\alpha}{2}}} d u\right)\right] \\
& \cdot \exp \left(-\mu T r^{\alpha} \sigma^{2}\right) d r .
\end{aligned}
$$

This completes the proof.

\subsubsection{Success probability for dynamic sleep}

From Sec. 4.2, we learned the load-awareness dynamic sleep mode operating principle. Here, a load level $x$ and a load level threshold $x_{0}$ are used to determine the next operation state for each SBS. That is to say, for any small cell with load level $x$, the small cell is active with probability $s(x)$, i.e. it is actually running with probability $s(x)$. Therefore, we can model the dynamic sleep as a functions $s(x)$, which implies that if the load level is measured automatically by the SBS is $x$, then it operates with probability $s(x)$ and sleeps with probability $1-s(x)$. Then, we defined the function $s(x)$ as the operation probability. And we find that the function $s(x)$ increases monotonically till the load level achieves the load level threshold value $x_{0}$.

In (10), via replacing $q$ by $s(x)$, the success probability $q_{s}^{\mathrm{DS}}$ for dynamic sleep mode can be given by

$$
\begin{aligned}
q_{s}^{\mathrm{DS}}= & 2 \pi \lambda_{s} s(x) \int_{r>0} r \exp \left[-\pi \lambda_{s} s(x) r^{2}\left(1+T^{\frac{2}{\alpha}} \int_{T^{-\frac{2}{\alpha}>0}} \frac{1}{1+u^{\frac{\alpha}{2}}} d u\right)\right] \\
& \times \exp \left(-\mu T r^{\alpha} \sigma^{2}\right) d r .
\end{aligned}
$$

The derivation process of the coverage probability $q_{s}^{\mathrm{DS}}$ for dynamic sleep is parallel to the one derived above for random sleep, just replacing with $s(x)$. Thus, the 
corresponding derivation process is no longer given, as an exercise for the readers. As a result, the success probabilities for the proposed random sleep mode and dynamic sleep mode can be given by Eqs. (10) and (19), respectively.

\subsection{Energy consumption minimization}

Energy consumption optimization and power efficiency are elaborated for the proposed two sleep modes.

\subsubsection{Energy consumption minimization for random sleep}

In random sleep, the success probability $q_{s}^{\mathrm{RS}}$ can be treated as a limitation to energy consumption minimization. Therefore, energy consumption minimization problem for random sleep is formulated as the following.

$$
\begin{gathered}
\min E_{\mathrm{RS}}=\sum_{i=0}^{\frac{\lambda_{s}}{\lambda_{m}}}\left\{\left[q_{\text {on }}^{i}\left(P_{\text {on }}+\beta P_{T}\right)+q_{\text {sleep }}^{i} P_{\text {sleep }}\right] t\right\}, \\
q_{S}^{\mathrm{RS}} \geq \varphi \\
\text { s.t. } \quad 0 \leq q_{\text {on }}^{i}, q_{\text {sleep }}^{i} \leq 1, \\
q_{\text {on }}^{i}+q_{\text {sleep }}^{i}=1
\end{gathered}
$$

where $\varphi$ is a constant which can be interpreted as QoS. The parameter $t$ is the measuring time of power consumption in the SBS.

Lemma 1. The success probability $q_{s}^{\mathrm{RS}}$ increases with increasing of the operation probability $q$, and so does total energy consumption $E_{\mathrm{RS}}$.

Proof. Let $x=r^{2}$, the success probability $q_{s}^{\mathrm{RS}}(q)$ with operation probability $q$ can be written as

$$
\begin{aligned}
q_{S}^{\mathrm{RS}}(q) & =2 \pi \lambda_{s} q \int_{r>0} r \exp \left(-a_{1} q r^{2}\right) \exp \left(-a_{2} r^{\alpha}\right) d r \\
& =2 \pi \lambda_{s} q \int_{x>0} \exp \left(-a_{1} q x\right) \exp \left(-a_{2} x^{\frac{\alpha}{2}}\right) d x
\end{aligned}
$$

Suppose operation probability $q^{*}>q$ and $x=\left(\frac{q}{q^{*}}\right) y, q_{s}^{\mathrm{RS}}(q)$ with operation probability $q^{*}$ can be expressed as

$$
\begin{aligned}
q_{S}^{\mathrm{RS}}\left(q^{*}\right) & =2 \pi \lambda_{s} q^{*} \int_{x>0} \exp \left(-a_{1} q^{*} x\right) \exp \left(-a_{2} x^{\frac{\alpha}{2}}\right) d x \\
& =2 \pi \lambda_{s} q \int_{y>0} \exp \left(-a_{1} q y\right) \exp \left[-a_{2}\left(\frac{q}{q^{*}}\right)^{\frac{\alpha}{2}} y^{\frac{\alpha}{2}}\right] d y .
\end{aligned}
$$

We have $\exp \left[-a_{2}\left(\frac{q}{q^{*}}\right)^{\frac{\alpha}{2}} y^{\frac{\alpha}{2}}\right]>\exp \left(-a_{2} y^{\frac{\alpha}{2}}\right)$ since $q^{*}>q$ and $\alpha>2$. Therefore, we can arrive at a conclusion that $q_{s}^{\mathrm{RS}}\left(q^{*}\right)>q_{s}^{\mathrm{RS}}(q)$ is true.

Therefore, energy consumption minimization occurs when $q_{s}^{\mathrm{RS}}$ satisfies the constraint tightly. 


\subsubsection{Energy consumption minimization for dynamic sleep}

Likewise, energy consumed by the SBS tier in dynamic sleep can be also minimized by means of the success probability $q_{s}^{\mathrm{DS}}$. Hence, energy consumption minimization in the dynamic sleep is formulated as

$$
\begin{gathered}
\min E_{\mathrm{DS}}=\sum_{i=0}^{\frac{\lambda_{s}}{\lambda_{m}}}\left\{\left[s\left(x_{i}\right)\left(P_{\mathrm{on}}+\beta P_{T}\right)+\left(1-s\left(x_{i}\right)\right) P_{\text {sleep }}\right] t\right\}, \\
q_{s}^{\mathrm{DS}} \geq \varphi \\
\text { s.t. } \quad s\left(x_{i}\right) \in[0,1] \\
\\
x_{i} \geq 0 .
\end{gathered}
$$

Using similar proof process derived in the proof for random sleep, $q_{s}^{\mathrm{DS}}$ is also an increasing function of load level $x$ based on the fact that $s(x)$ keeps increasing when $x \rightarrow x_{0}$. Hence, the optimal operation probability bringing about energy consumption minimization for the dynamic sleep can be identified when $q_{s}^{\mathrm{DS}}=\varphi$.

\section{Simulation Results}

In this section, we evaluate the performance of the proposed energy saving strategy for the co-channel deployment HSCN. The spatial distribution for SBSs deployed in the MBS coverage range is modeled as HPPP. ${ }^{4}$ In random sleep, state transition of a SBS between waking and sleeping is deemed as a binomial distribution event. While, under the dynamic sleep mode, the SBS operation relies on the measured load level $x$ and the predefined threshold $x_{0}$. The parameters used for simulation are summarized in Table 2.

In the random sleep, the state transitions for a SBS between awake or sleeping is deemed as a binary event. Therefore, the operation probability $q_{\text {on }}$ on this occasion takes value from $[0,1]$.

On the other hand, the operation of a small cell will totally up to its load level $x$ and threshold $x_{0}$ when the dynamic sleep is put to use in the small cell tier. In other words, if the SBS has load level $x \in\left(0, x_{0}\right)$, it operates with probability $s(x)$ and

\begin{tabular}{lc} 
Table 2. & Parameters used in simulation. \\
\hline Parameter & Value \\
\hline$\alpha$ & 4 \\
$\lambda_{s}$ & 0.1 \\
$\lambda_{m}$ & 0.001 \\
$t$ & $365 \times 24 \times 60 \times 60 \mathrm{~s}$ \\
$\mu$ & 0.001 \\
$T$ & $-10 \mathrm{~dB}$ \\
$n$ & 1 \\
Carrier frequency & $2.6 \mathrm{GHz}$ \\
Bandwidth & $20 \mathrm{MHz}$ \\
MU traffic model & Wrap around \\
\hline
\end{tabular}




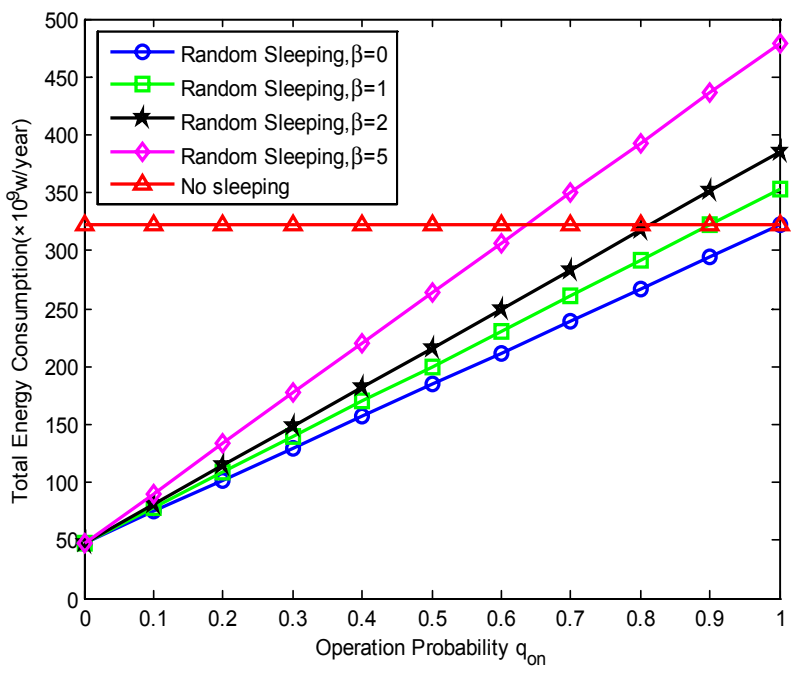

Fig. 3. Total energy consumption for random sleep.

sleeps with probability $1-s(x)$. Hence, several positive integers are assigned to the load level threshold $x_{0}$ which is also used to act as the value space of load level $x$.

Figure 3 shows the changing of total energy consumption for the proposed random sleep when the SBS operation probability $q_{\text {on }}$ varies. For a given power control parameter $\beta$, growing energy is consumed by the SBS tier with the increasing of $q_{\text {on }}$. The main reason is that more SBSs will be more inclined to be active with the rise of $q_{\text {on }}$. It can be also seen that total energy the SBS tier consumes under the random sleep is lesser than that under no sleep within a certain $q_{\text {on }}$ scope. This directly goes to show the effectiveness of the proposed random sleep. And increasing energy is consumed with the larger $\beta$. Thus, $\beta$ effectively influences the performance on the total energy consumption. This can be explained that the power consumed by an awaked SBS applying power control is larger than the power saved by its sleeping neighbors. On the other hand, do not blindly configure operation probability (or sleep probability) and power control parameter $\beta$ for small cell tier when applying random sleep in it, on the contrary, lesser energy consumption with proper parameter settings could be reachable, as reflected by Fig. 3. For example, let us suppose random sleep with power control parameter $\beta=5$ is used in the small cell tier, the operation probability $q_{\text {on }}$ must be configured between 0 and 0.63 in consideration of the energy saving. Otherwise, the energy consumption under this sleep mode will exceed that without sleeping.

Figure 4 illustrates that the relation between the load level $x$ and the total energy consumed by the small cell tier applying no sleeping or dynamic sleeping with the different load level thresholds $x_{0}$ which is used by every SBS to determine its next step of running state. For a given load level $x_{0}$, the first sight is the total power consumption goes up with the increase of the load level $x$ when applying 


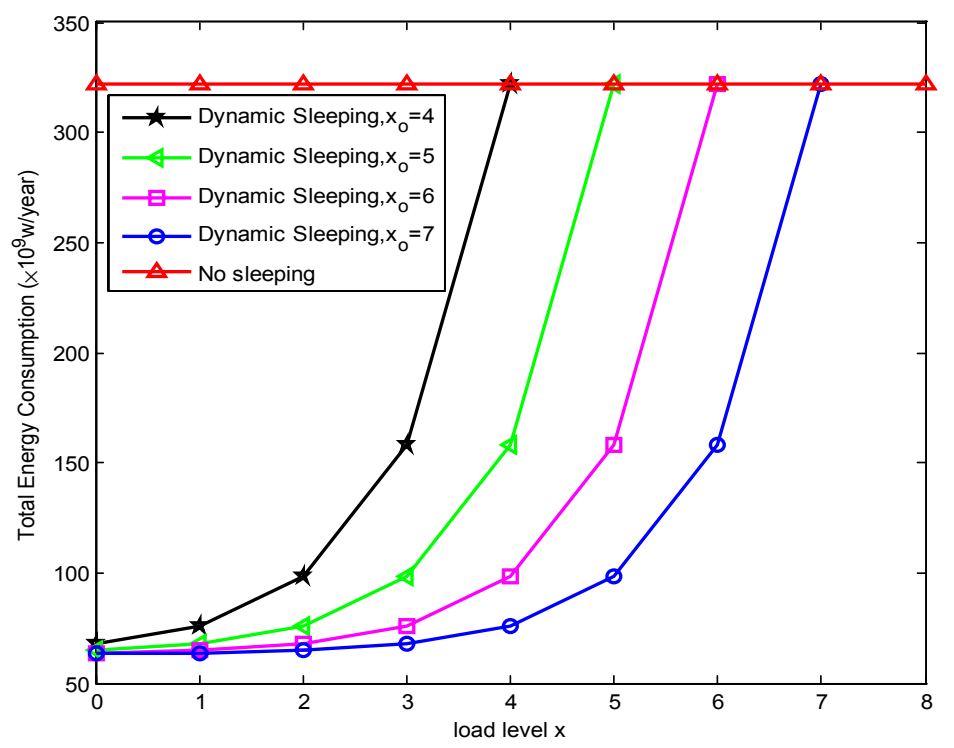

Fig. 4. Total power consumption for dynamic sleep with different $x_{0}$.

dynamic sleep. The reason behind this is that the small cell base station tends to be more active when its load level $x$ gets closer to its preset load level threshold $x_{0}$. Also, it can be observed that the dynamic sleep mode we proposed in the previous sections consumes less energy, compared to the same situation with no sleep. Similar to Fig. 1, the simulation result demonstrates dynamic sleep is really effective on the purpose of saving energy. For example, for this case whose load level threshold $x_{0}$ is 7 , lesser and lesser energy is consumed by the small cell tier when the load level $x<7$. This is mainly because the SBS operates with probability as long as the load level $x$ satisfies $x<x_{0}$. However, once its load level $x$ surpasses threshold $x_{0}$, the SBS switches on with certainty. Therefore, at this point, the energy consumed by the SBS starts equating with that under no sleep. And Fig. 4 can be also seen that the smaller the load level threshold $x_{0}$ is, the greater the energy consumed by the small cell tier. This can be interpreted as that the SBS is more likely to become easier active when its load level threshold $x_{0}$ is assigned a lower value. Finally, we can understand that a proper setting of the load level threshold $x_{0}$ for the SBS is of crucial importance to its outstanding performance on saving energy.

Figure 5 presents how the total energy consumption changes when employing the proposed dynamic sleep in the SBS tier. As a matter of convenience, the load level threshold $x_{0}=6$ is taken. Primarily, total energy consumption rises with the increase of load level $x$ as well as power control parameter $\beta$. It can be found that dynamic sleep with $\beta>0$ exceeds no sleep as $x$ comes closer to the predefined $x_{0}$, for instance when $x$ is larger than 5 . This is because that the power consumed by an awaked SBS employing the power control is larger than power saved by a sleeping SBS. 


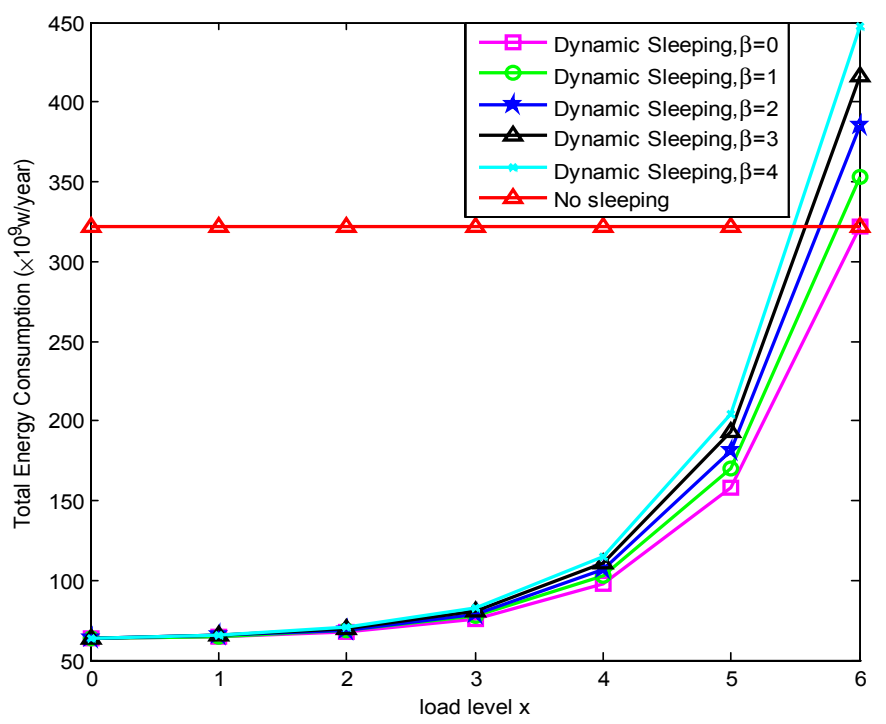

Fig. 5. Total power consumption for dynamic sleep.

Meanwhile, SBSs tend to be more and more active. However, for the vast majority of $x \in\left(0, x_{0}\right)$, clearly, the total energy consumption under dynamic sleep is far below that under no sleep. It thus proves that the proposed dynamic sleep is an effective approach which can be used in the SBS tier to save considerable energy. Comparing Fig. 3 with Fig. 5, dynamic sleep consumes less energy than random sleep as indicated by Fig. 4. This comparison also directly answers which the proposed sleep mode is more effective on energy conservation.

Thus far, it could well be that dynamic sleep which can be treated as load aware operation mechanism have far superior potential than random sleep on energy saving. Next, we will go further to clarify the superiority of one sleep mode over another from the coverage probability to power efficiency, not only from energy saving.

Figure 6 illustrates the success probability versus AWGN power $\sigma^{2}$ for the proposed sleep modes. It is observed that the success probability after applying dynamic sleep model is slightly better than the case without sleep strategy and far better than random sleep. This is due to the fact that the small cells are able to adjust their energy saving strategy, i.e. keep active or idle, according to the varying of traffic load when using dynamic sleep mode. In addition, this figure shows success probability almost has no improvement when AWGN is fairly significant, when comparing dynamic sleep with no sleep, even it becomes quite low when $\sigma^{2} \rightarrow 0$. This implies that the existence of AWGN greatly poses negative influence on the success probability.

Figure 7 presents the power efficiency under various noise powers when applying no sleeping, random sleeping and the proposed dynamic sleeping strategies. Power efficiency can be obtained through power consumption divided by success probability. By applying sleeping modes, the power efficiency can be significantly improved. 


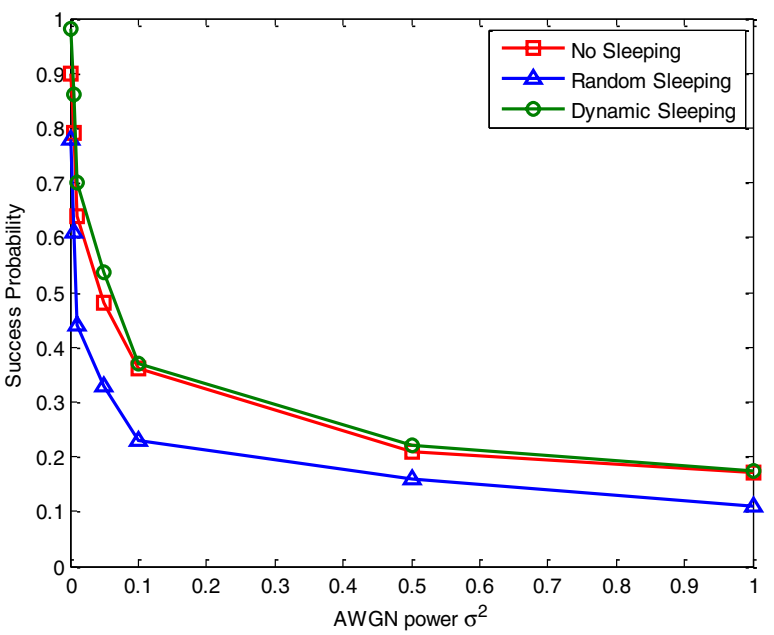

Fig. 6. Success probability for sleep modes.

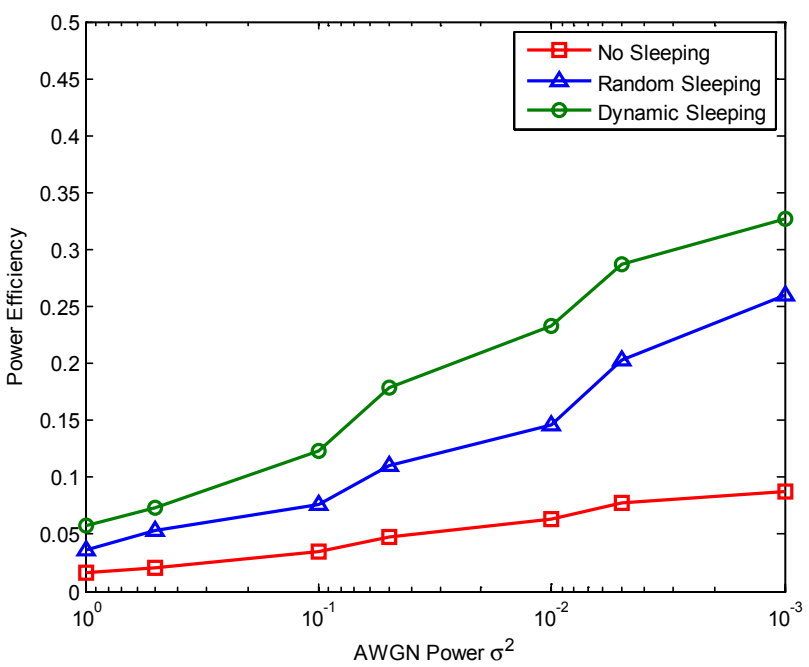

Fig. 7. Power efficiency for sleep modes.

For instance, the power efficiency is 0.025 when noise power is 0.1 , while the power efficiency can exceed 0.05 and 0.1 by using random sleeping and dynamic sleeping, respectively. In addition, the power efficiency in dynamic sleep outperforms that in random sleep, especially in the case of large noise power. In a word, the simulation results reveal that the proposed load-awareness dynamic sleep is superior to the proposed random sleep in terms of energy saving, success probability and power efficiency. 
In summary, from Figs. 3 to 7, we demonstrate that the proposed two different sleep modes are both practicably effective methods on reducing energy consumption as well as improving coverage probability and power efficiency. And it is clearly verified that dynamic sleep have more gain in certain respects, such as energy saving, coverage probability and power efficiency than the random sleep. This is the result we are more willing to see because the dynamic sleep can be regarded as a load aware power saving mechanism.

\section{Conclusions}

In this study, we investigate energy efficient small cells by devising energy saving strategies. In order to reduce energy consumption and improve power efficiency, random sleep mode and load-awareness dynamic sleep mode are proposed, where the former relies on the basis of binomial distribution of SBS operation probability, the latter depends on the traffic load level of SBSs. The closed-form expression of success probability is derived for each of the proposed sleep modes. Energy consumption minimization problems are formulated under the success probabilities constraint. Based on the analysis and simulation results, we demonstrate that the proposed two different sleep modes are both practicably effective methods on reducing energy consumption as well as improving coverage probability and power efficiency. Moreover, it is clearly verified that dynamic sleep have more gain in certain respects, such as energy saving, coverage probability and power efficiency than the random sleep. This is the result we are more willing to see because the dynamic sleep can be regarded as a load aware power saving mechanism. In the future research work, we will jointly consider resource allocation and access control with our energy saving method in order to further study the energy issues in small cell networks.

\section{Acknowledgments}

This research is supported in part by the National Natural Science Foundation of China (Grants Nos. 61301148, 61272061 and 71403299), the Hunan Natural Science Foundation of China (Grants No. 2016JJ3041) and the open fund of State Key Laboratory of Geo-information Engineering (Grants No. SKLGIE2016-M-4-2).

\section{References}

1. F. Ashraf and L. Ho, Sleep mode techniques for small cell deployments, IEEE Commun. Mag. 49 (2011) 72-79.

2. A. Azad, S. Alouf, E. Altman, V. Borkar and G. Paschos, Optimal control of sleep periods for wireless terminals, IEEE J. Sel. Areas Commun. 29 (2011) 1605-1617.

3. Cisco, Cisco visual network index: Global mobile data traffic forecast update, 2015-2020, http://www.cisco.com/c/en/us/solutions/collateral/service-provider/visual-networking-index-vni/mobile-white-paper-c11-520862.pdf, February (2016).

4. S. G. Foss and S. A. Zuyev, On a Voronoi aggregative process related to a bivariate poisson process, Adv. Appl. Probab. 28 (1996) 965-981. 
5. X. Ge, S. Tu, T. Han, Q. Li and G. Mao, Energy efficiency of small cell backhaul networks based on GaussMarkov mobile models, IEEE Access 3 (2015) 158-167.

6. W. Guo and Tim O'Farrell, Green cellular network: Deployment solutions, sensitivity and tradeoffs, in Proc. 2011 Wireless Advanced (WiAd), London, England, June (2011), pp. $42-47$.

7. A. Gupta and R. K. Jha, Fernandez-Duran, A survey of 5G network: Architecture and emerging technologies, IET Netw. 4 (2015) 1206-1232.

8. K. Li, X. Tang and K. Li, Energy-efficient stochastic task scheduling on heterogeneous computing systems, IEEE Trans. Parallel Distrib. Syst. 25 (2014) 2867-2876.

9. K. Zou, K. Yang, M. Wang, B. Ren, J. Hu, J. Zhang, M. Hua and X. You, Network synchronization for dense small cell networks, IEEE Wirel. Commun. 22 (2015) 108-117.

10. T. Li, Z. Xiao, H. M. Georges et al., Performance analysis of co-and cross-tier device-todevice communication underlaying macro-small cell wireless networks, KSII Trans. Internet Inf. Syst. 10 (2016) 1481-1500.

11. W. Li, W. Zheng, Y. Xie and X. Wen, Clustering based power saving algorithm for selforganized sleep mode in femtocell networks, Proc. 15th Int. Symp. Wireless Personal Multimedia Communications (WPMC), Taipei, China, September (2012), pp. 379-383.

12. Z. Longxin, L. Kenli, Z. Fan and L. Keqin, Maximizing reliability with energy conservation for parallel task scheduling in a heterogeneous cluster, Inf. Sci. 319 (2015) $113-131$.

13. Qualcomm, 1000x mobile data challenge, https://www.qualcomm.com/documents/ 1000x-mobile-data-challenge, November (2013).

14. Qualcomm, LTE heterogeneous networks, https://www.qualcomm.com/documents/lteheterogeneous-networks, July (2013).

15. S. Samarakoon, M. Bennis, M. W. Saad and M. Latva-aho, Opportunistic sleep mode strategies in wireless small cell networks, in Proc. 2014 IEEE Int. Conf. Communications (ICC), Sydney, NSW, June (2014), pp. 2707-2712.

16. S. Wu, C. Chen and M. Chen, Collaborative wakeup in clustered adhoc networks, IEEE J. Sel. Areas Commun. 29 (2011) 1585-1594.

17. Z. Xiao, T. Li, W. Ding et al., Dynamic PCI allocation on avoiding handover confusion via cell status prediction in LTE heterogeneous small cell networks, Wirel. Commun. Mob. Comput. 16 (2016) 1972-1986.

18. Z. Xiao, H. Liu, V. Havyarimana et al., Analytical study on multi-tier $5 \mathrm{G}$ heterogeneous small cell networks: Coverage performance and energy efficiency, Sensors 16 (2016) 1854.

19. K. Xie, J. Cao, X. Wang et al., Optimal resource allocation for reliable and energy efficient cooperative communications, IEEE Trans. Wirel. Commun. 12 (2013) 49945007.

20. K. Xie, X. Wang, X. Liu et al., Interference-aware cooperative communication in multiradio multi-channel wireless networks, IEEE Trans. Comput. 65 (2016) 1528-1542.

21. W. Yan, L. Kenli, C. Hao and L. Keqin, Energy-aware data allocation and task scheduling on heterogeneous multiprocessor systems with time constraints, IEEE Trans. Emerg. Top. Comput. 2 (2014) 134-148.

22. X. Yuming, L. Kenli and L. Keqin, A genetic algorithm for task scheduling on heterogeneous computing systems using multiple priority queues, Inf. Sci. 270 (2014) $255-287$.

23. X. Yuming, L. Kenli and L. Keqin, A hybrid chemical reaction optimization scheme for task scheduling on heterogeneous computing systems, IEEE Trans. Parallel Distrib. Syst. 26 (2015) 3208-3222. 
24. 3GPP, Small cell enhancements for E-UTRA and E-UTRAN physical layer aspects (Release 12). TR 36.872, http://www.3gpp.org/DynaReport/36872.htm, May (2013).

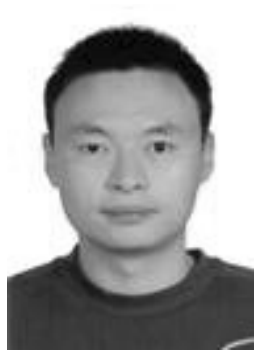

Zhu Xiao received his M.S. degree in Communication and Information System from Xidian University, China, in 2007. He joined the State Key Laboratory of Integrated Services Networks (ISN) at Xidian University in 2007 and obtained his Ph.D in December 2009. Currently, he is an Associate Professor at the College of Computer Science and Electronic Engineering, Hunan University, China. His research interests include wireless/mobile communications, wireless localization, next-generation communications and heterogeneous networks.

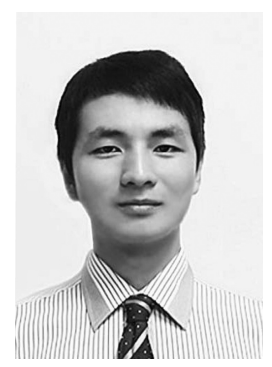

Shuangchun Li received his B.S. degree in Information Science from Hunan University, China, in 2015. He is currently pursuing his M.S. degree in Communication and Information System at Hunan University, His interests focus on power and resource management in future wireless and cellular networks.

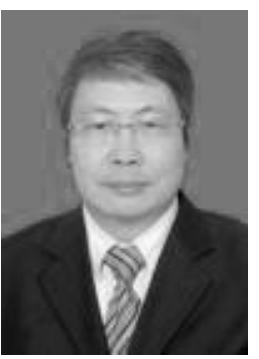

Xiaochun Chen received his B.S. degree in Economy from Hunan University of Finance and Economics in 1983, and his M.S. degree and Ph.D in Economy from Kurume University, Japan, in 1996 and 1999, respectively. Currently, he is a professor and Ph.D. supervisor at Hunan University, China.

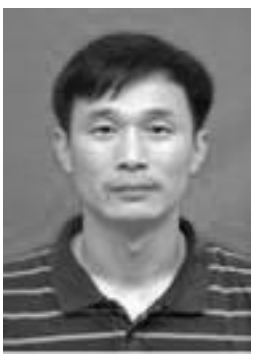

Dong Wang received his B.S. degree and Ph.D. in Computer Science from Hunan University in 1986 and 2006, respectively. From December 2004 to December 2005, he was a visiting scholar at University of Technology, Sydney, Australia. Since 1986, he has been working at Hunan University, China. Currently, he is a professor at Hunan University. His main research interests include network test and performance evaluation, wireless communications and mobile computing.

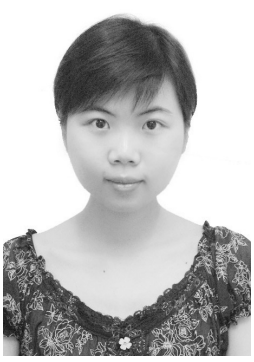

Wenjie Chen received her M.S degree in Management from Hunan University, China, in 2009. She obtained her Ph.D in Business Administration from Hunan University, China, in December 2013. Since 2014, she has been a lecturer with the Business College, Central South University of Forestry and Technology, China. Her research interests include low-carbon and energy saving technology, internet of vehicles and innovation networks. 